\title{
Study of blades inclination influence of gate impeller with a non-Newtonian fluid of Bingham
}

\author{
Lakhdar Rahmani $^{1}$, O. Seghier ${ }^{1}$, B. Draoui ${ }^{1}$ and E. Benachour ${ }^{1}$ \\ ${ }^{1}$ Arids zones energetic laboratory (ENERGARID), TAHRI Mohamed University of Béchar, BP 417, 08000 Béchar, Algeria.
}

\begin{abstract}
A large number of chemical operations, biochemical or petrochemical industry is very depending on the rheological fluids nature. In this work, we study the case of highly viscous of viscoplastic fluids in a classical system of agitation: a cylindrical tank with plate bottom without obstacles agitated by gate impeller agitator. We are interested to the laminar, incompressible and isothermal flows. We devote to a numerical approach carried out using an industrial code CFD Fluent 6.3.26 based on the method of finites volumes discretization of Navier - Stokes equations formulated in variables (U.V.P). The threshold of flow related to the viscoplastic behavior is modeled by a theoretical law of Bingham. The results obtained are used to compare between the five configurations suggested of power consumption. We study the influence of inertia by the variation of Reynolds number.
\end{abstract}

\section{Introduction}

Usually many industrial processes often face difficulties related to the implementation of non-Newtonian fluids such as polymer solutions, detergents, petroleum products, biological fluids, food, drilling fluids and emulsions to be transported, stirred and mixed. If agitation is intended to promote a physical process, such as homogenization or enhancement of heat transfer, the prediction of the power necessary for its implementation will be the primary concern. We have studied the hydrodynamics developed in an agitation in the presence of viscoplastic fluids. Hydrodynamics is a key control and process optimization. His knowledge and control are crucial to improve efficiency. To do this, we seek to determine the influence of rheological properties of the fluid on these flows.

The objective of this work is the study of mechanical agitation of highly viscous fluid by an agitator-type proximity barrier using five configurations depending on the inclination angles of blades with a non baffled cylindrical tank with flat bottom such that the geometry is close to an industrial setting. The choice of this geometry has the advantage of generating a flow that can, in first approximation considered as a two-dimensional. The flow regimes studied are laminar and implemented fluids are viscoplastic type of Bingham. The difficulty of modeling problems lies in the viscoplastic behavior of the fluid. The results enable a comparative study between five blade configurations chosen especially from the point of view of energy consumption (number of power), this allows us to make a choice of an optimal configuration (i.e.) with low energy consumption.

\section{Numerical models}

The mixing systems are presented in Figure 1. The tank is a flat-bottomed vessel (inside diameter: D) equipped with a two blades impellers (diameter $\mathrm{d}$ ). $\mathrm{H}$ is the fluid height. Values for the impellers width L, agitator-to-wall clearance $\mathrm{w}$ and impairs thickness e, Alpha is the inclination of the agitator blades are reported in Table 1 .

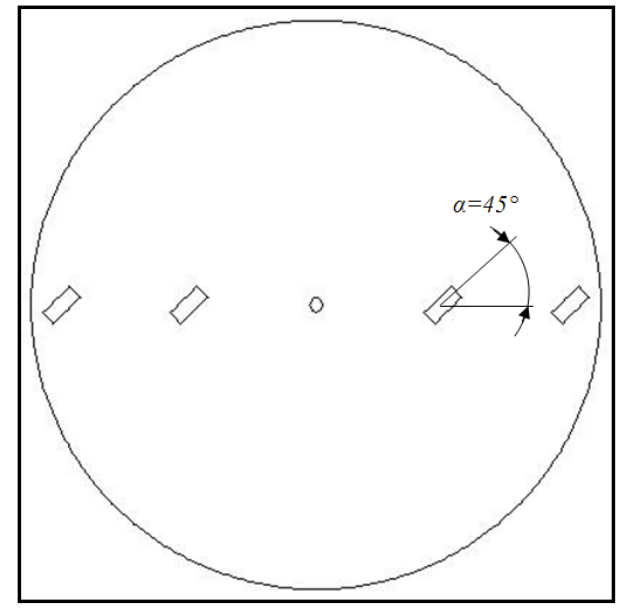

Figure 1. The mixing system model.

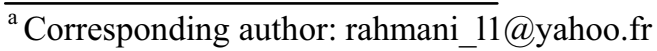


Table 1. Geometrical characteristics of agitator.

\begin{tabular}{|c|c|}
\hline $\mathbf{D}$ & $\mathbf{1}$ \\
\hline $\mathrm{d}$ & 0.96 \\
\hline $\mathrm{da}$ & 0.023 \\
\hline $\mathrm{E}$ & 0.027 \\
\hline 1 & 0.067 \\
\hline $\mathrm{w}$ & 0.02 \\
\hline$\alpha$ & $\begin{array}{r}0^{\circ}, 15^{\circ}, 30^{\circ} \\
45^{\circ}, 60^{\circ}\end{array}$ \\
\hline
\end{tabular}

\section{Comportment law of fluid}

To study the yield stress fluids which constitute the purpose of this paper, mixing systems are dealt with a numerical simulation using Viscoplastic fluids modeled by Bingham law:

$$
\begin{aligned}
& \overline{\bar{D}}=0 \text { for }\|\tau\|<\tau_{0} \\
& \overline{\bar{\tau}}=\left(\frac{\tau_{0}}{\dot{\gamma}}+\eta_{\infty}\right) \overline{\bar{D}} \text { for }\|\overline{\bar{\tau}}\|>\tau_{0}
\end{aligned}
$$

Where $\overline{\bar{D}}$ and $\bar{\tau}$ are, respectively, the rate of strain tensor and the stress tensor. $\tau_{0}$ is the yield stress.

The shear rate is defined as $\dot{\gamma}=\sqrt{2 \operatorname{tr} \overline{\bar{D}}^{2}}$ where tr stands for the trace.

$$
\overline{\bar{D}} \text { And } \bar{\tau} \text { are defined as: } \overline{\bar{D}}=1 / 2\left(\nabla V+\nabla V^{T}\right)
$$$$
|\bar{\tau}|=\sqrt{\frac{1}{2} \tau: \bar{\tau}}
$$

According to equations (1) and (2), the flow domain for Bingham fluid is characterized by two distinct regions. In the regions where $|\overline{\bar{\tau}}| \prec \tau_{0}$ the material behaves as a solid (rigid zone), and in the regions where $|\bar{\tau}| \succ \tau_{0}$ the material flow with an apparent viscosity $\eta_{a p}$

$$
\eta_{a p}=\eta_{\infty}+\frac{\tau_{0}}{\dot{\gamma}}
$$

The major difficulty with the constitutive equation (1) and (2) when used for numerical simulation is the discontinuity associated with infinite value of the viscosity when $|\tau|$ approaches $\tau_{0}$. This is reached closed to the yield surface delimiting plug regions. Some numerical works have been published on methods adapted to the initial set of equations [(1), (2)] completed by continuity equation and momentum equation in stress formulation. But most of the published works on this topic use modified versions which consist in replacing equations. (1) + (2) By a unique and continuous equation written with a variable viscosity $\eta_{a p}[9]$ :

$$
\tau=2 \eta_{a p} \overline{\bar{D}}
$$

\section{Model equations and computational procedure}

The equations describing fluid flow are derived from the conservation of mass and momentum which are also known as Navier-Stokes equations for fluids of constant physical properties:

$$
\begin{gathered}
\nabla \cdot V=0 \\
\rho \frac{\partial V}{\partial t}+\rho(V . \nabla)-\eta_{a p} \Delta V-2 \overline{\bar{D}} \cdot \nabla \eta_{a p}+\nabla P=0
\end{gathered}
$$

\subsection{Boundary conditions}

In the CFD code used two reference frames can be treated. One is stationary, the impeller is in rotation and the tank is fixed. The other frame, which is rotating, the impeller is kept stationary while the outer wall of the vessel is given an angular velocity equal and opposite to the velocity of the rotating frame. Constant boundary conditions have been set respecting a fixed reference frame (RRF) approach. The boundary conditions for velocity are fixed on the gate impeller and the vessel. On the gate impeller: Moving reference frame implemented in the CFD code.

Flow regime must be retained in non-Newtonian laminar flow, it can be used to define a generalized Reynolds number. From this generalized Reynolds number, an apparent viscosity nap can be deduced. Therefore, nap is defined as the viscosity of the Newtonian fluid providing the same power consumption as the non-Newtonian fluid for the studied system:

$$
\operatorname{Re} g=\frac{\rho \cdot N \cdot d^{2}}{\eta_{a p}}
$$

Metzner and Otto [14] introduced the concept of apparent viscosity which is linked to an apparent shear rate $\dot{\gamma}_{a p}$ using the rheological characterization of the fluid $\eta\left(\dot{\gamma}_{a p}\right)$. They suggested this apparent shear rate to be proportional to the rotation frequency $\mathrm{N}$ :

$$
\dot{\gamma}_{a p}=\mathrm{Ks} . \mathrm{N}
$$

These results for the bi-viscosity model are quite similar for $\gamma_{c}=10^{-2}$. 


\subsection{Validation}

To verify the model of the fluid and to validate our results we compared our power number with previous works on an anchor agitator. Figure 2 gives an example of comparison between our results and a numerical work of Marouche [8]. The results show a satisfactory agreement.

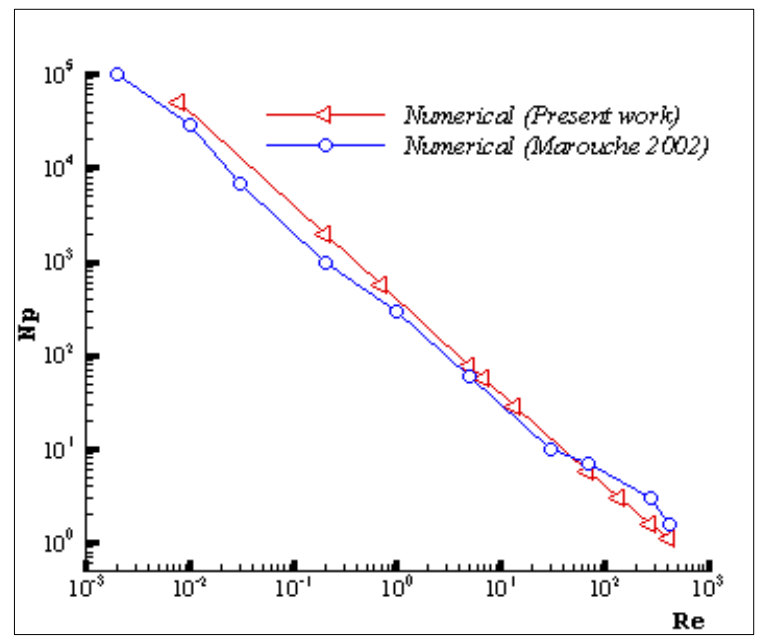

Figure 2. Variation of power number as function of Reynolds number.

\section{Results and interpretations}

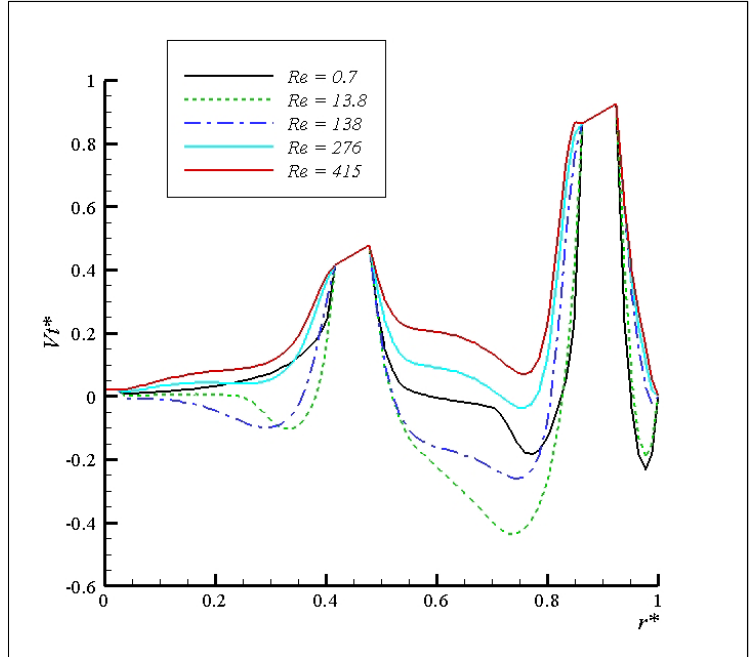

Figure 3. Influence of inertia on the tangential velocity at the blade and extension.

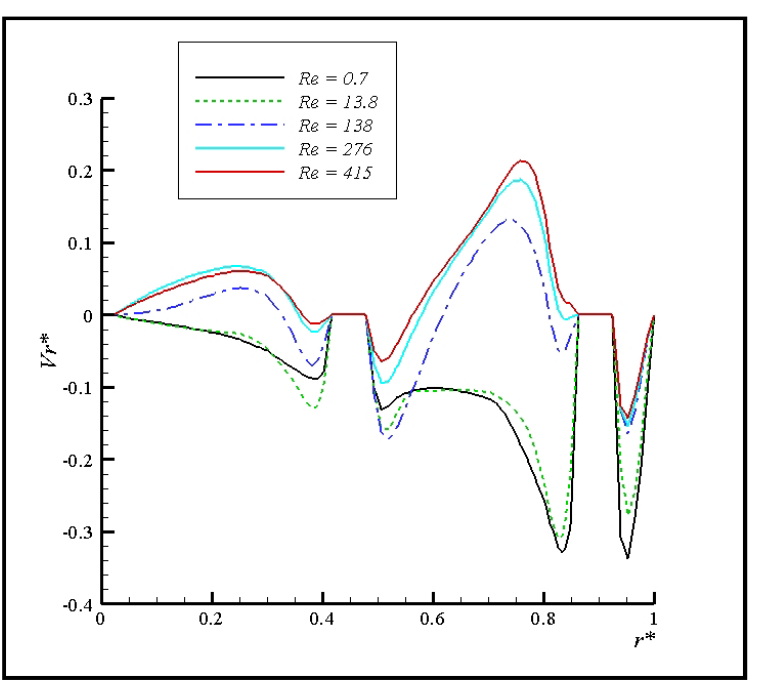

Figure 4. Influence of inertia on the radial velocity at the blade and extension.

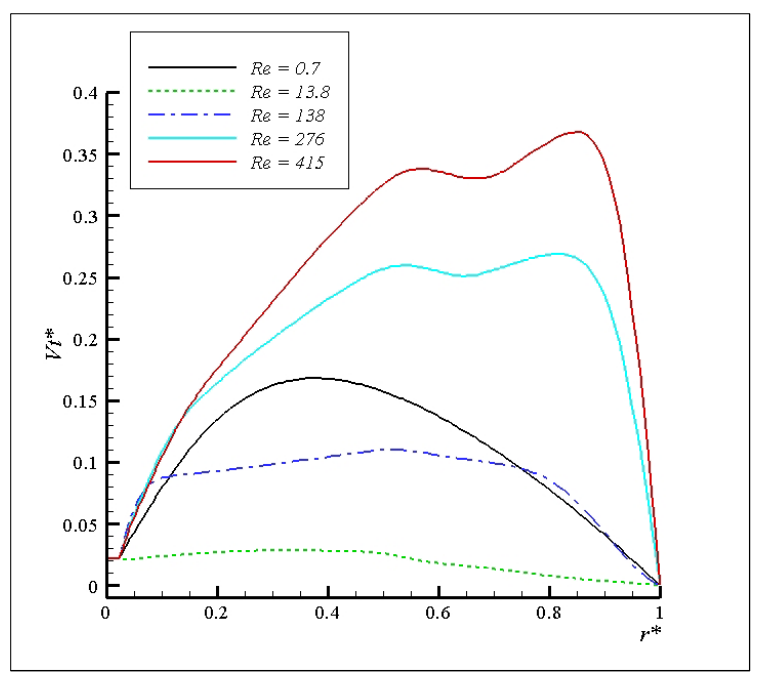

Figure 5. Influence of inertia on the tangential velocity at the mediator.

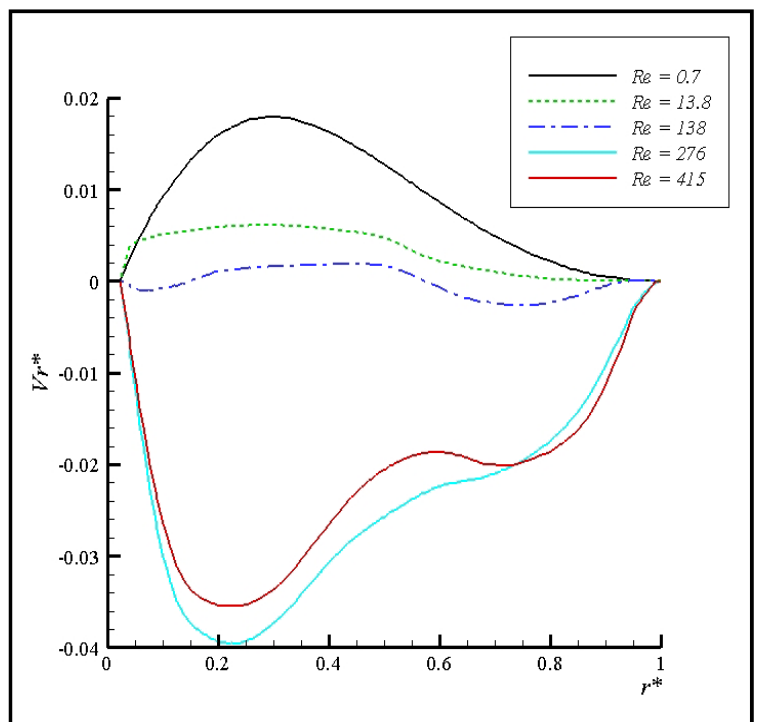

Figure 6. Influence of inertia on the radial velocity of the mediator. 


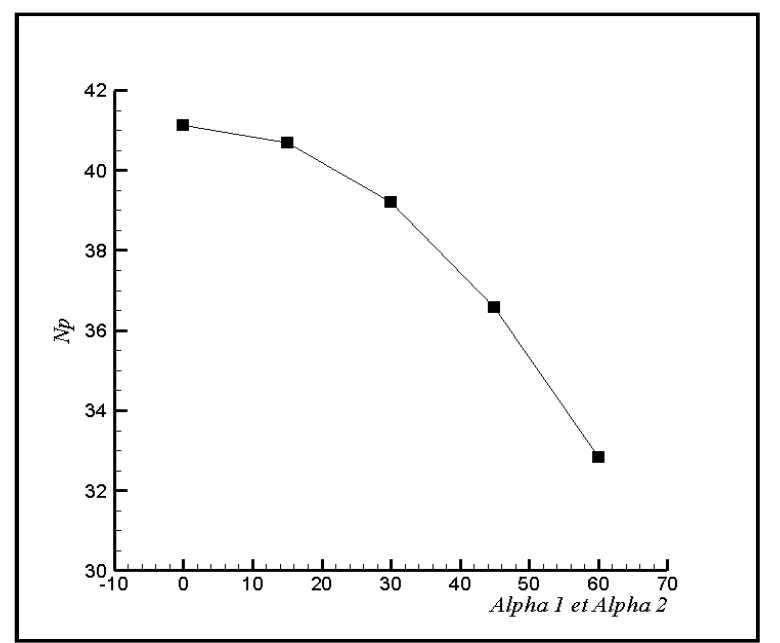

Figure 7. Inclination influence of the two blades on the power consumption.

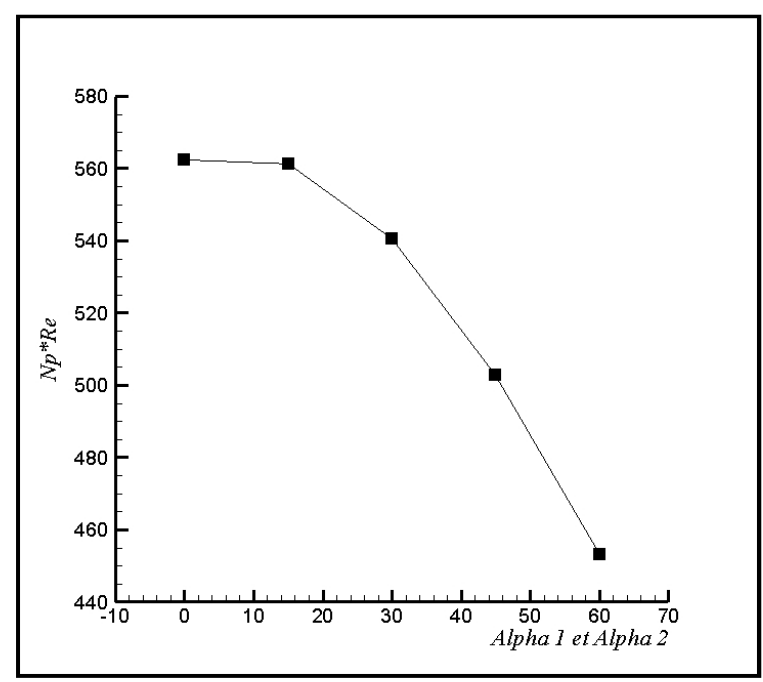

Figure 8. Inclination influence of the two blades on the Product $\mathrm{Np} * \mathrm{Re}$.

Based on the analysis of previous curves results which show the variation in energy consumption compared to the blade pitch position or one by one or both at the same time, we find that the optimal configuration that consumes less energy is the fifth configuration (i.e.) configuration where Alpha 1 and Alpha 2 equals $60^{\circ}$.

Figures 3, 4, 5 and 6 show the variation of the tangential and radial velocity of blade and its extension and on the mediator according to the radial position. We studied the influence of Reynolds number on the nature of the flow by varying this parameter from 0.7 to 415 for a Bingham fluid threshold $\tau 0=1 \mathrm{~Pa}$.

The tangential component of the velocity varies in the following manner Figure 3 growth of the tangential velocity from the agitator shaft to the first blade and a linear increase between the two ends thereof, a decrease to a relative minimum, and growth until the inner end of the second blade and a second linear increase in the level of the second blade, once end, a sudden decrease of velocity to the vessel wall which is zero. It is noted again that the tangential velocity increases with the increase of Reynolds number in the two zones, one between the axis of the agitator and the first vane and the other between the two blades.

We note that upstream of the first blade and the second, the tangential velocity $\mathrm{Vt}^{*}$ is negative for low Reynolds numbers, when $\mathrm{Re}$ is greater than 150, the tangential velocity is always positive, for against the radial velocity $\mathrm{Vr}^{*}$ Figure 5 is always positive: this means that the inner end of each blade draws fluid from the center to the wall of the vessel, while the outer end of the second blade has a tendency of the shaving of the tank towards the center thereof.

On the mediator Figure 4, the velocity is nearly zero for low Reynolds numbers, but reaches a maximum with increasing relative inertia. Moreover this maximum is lower than the outer end of the second blade, which means that the flow in this zone is delayed beyond this maximum speed decreases as one moves towards the wall of the vessel, although to a slope greater than that of the rising part of the curve.

The influence of Reynolds number on the profile of the radial velocity $\mathrm{Vr}^{*}$ on the blades Figure 5, we see that the variation of the radial velocity is noticeable Clearly between axis of the rod end and the internal blade and the first entre le two blades, this variation is Rapidly decreasing to a low Reynolds number, we noted the existence of negative velocities which results in the formation of recirculation area between the two blades and near the wall where the flow is delayed in this area. Moreover, on the mediator Figure 6, the radial velocity is zero Almost.

We note that the number of power varies linearly with the inertia $(\mathrm{Re})$ on a logarithmic scale, produced by the cons of $\mathrm{Np} * \mathrm{Re}$, the constant of laminar flow is $\operatorname{Re}<100$ equal to 452 for our mobile, All above this value, there is a rapid increase in the product $\mathrm{Np} * \mathrm{Re}$, which means that the power consumption increases faster than the Reynolds number.

\section{Conclusions}

This contribution focuses on the viscoplastic fluids, which behavior is poorly controlled and which intervene in many processes, particularly the polymerization in emulsion. During the latter, the behavior progresses through time: practically, Newtonian at the beginning of the reaction, then becomes shear thinning and finally viscoplastic as long as the concentrations increase. The hydrodynamics of viscoplastic fluids in complex geometries such as the systems of agitation is modestly approached. However it plays a significant role, in the control and the effectiveness of processing since it conditions the heat and mass transfer. The problem is analyzed under the numerical aspect. The latter is not treated by a specific code but using an existing industrial code which is the Fluent code. The numerical simulations carried out in geometries $2 \mathrm{D}$ made it possible to show the important significations by the viscoplastic behavior and in particular, the quasi immobilization of the fluids in a broad area when the effects of yield are dominant. It is worth nothing that this figure does not work if we 
consider the mixture of two substances of this type of fluid.

Characterization of laminar flow and power consumption in an agitated vessel with two blades agitators has been developed in order to provide a physical analysis of mixing in viscoplastic fluids.

Has led us to see that this type of fluid for the choice of the fifth configuration is appropriate and consumes less power compared to other configurations to be precise our simulation has shown that we can make a saving of $20 \%$, hence the interest to use it often in the case of these fluids.

\section{References}

1. K. Yapici, B. Karasozen, M. Schafer, Y. Uludag. Numerical investigation of the effect of the Rushton type turbine design factors on agitated tank flow characteristics. Chem. Eng. and Proc. (2007).

2. F. Ein-Mozaffari, C.P.J. Bennington, G.A. Dumont, Suspension yield stress and the dynamic response of agitated pulp chests. Chem. Eng. Sci. 60 (2005).

3. T.P. Elson, The growth of caverns formed around rotating impellers during the mixing of a yield stress fluid, Chem. Eng. 96 303-319 (1990).

4. D. Anne-Archard, M. Marouche, H.C. Boisson. Hydrodynamics and Metzner-Otto correlation in stirred vessels for yield stress fluids. Chem. Eng. J. 125, 15-24 (2006).

5. J.N. Haque, T. Mahmud, K. Roberts, Modeling turbulent flows with free surface in baffled agitated vessels, Ind. Eng. Chem. Res. 45, 2881-2891 (2006).

6. V.V. Ranade, S.M.S. Dommeti, Computational snapshot of flow generated by axial impellers in baffled stirred vessels, Trans. Inst. Chem. Eng. 74, 476-484 (1996).

7. J.J. Derksen, Numerical simulation of solid suspension in a stirred tank, AIChE J. 49, 27002714 (2003).

8. M. Marouche, Hydrodynamique d'un système d'agitation en fluide viscoplastique et en régime laminaire inertiel, $\mathrm{Ph}$. D Theses, National Polytechnic Institut of Toulouse. (2002).

9. L. Rahmani et al. Heat transfer to Bingham fluid during laminar flow in agitated tank, Int. Rev. Mech. Eng. 09, (2013).

10. E.J. O'Donovan, R.I. Tanner, Numerical analysis of the Bingham squeeze film problem, J. of Non-New. Flu. Mec. 15, 75-83, (1984).

11. S.V. Patankar. Numerical heat transfer and fluid flow. Mc-Graw Hill. (1980).

12. G.C. Vradis, M.V. Otugen, The axisymmetric sudden expansion flow of a non-Newtonian viscoplastic fluid, J. Fluid Eng. 119, 193-200 (1997).

13. M. Marouche, D. Anne-Archard, H.C. Boisson, A numerical model of yield stress fluid dynamics in a mixing vessel, Appl. Rheol. 12, 182-191 (2002).

14. A.B. Metzner, R.E. Otto, Agitation of nonNewtonian fluids, AIChE J. 3, 3-10 (1957). 